\title{
Delirium numa enfermaria de Medicina Interna - impacto na prática clínica
}

\author{
Delirium in internal medicine ward - impact on clinical practice
}

\author{
Filipa Anaia Quaresma ${ }^{1}$, Amélia Maria Lérias Duarte², Paulo Sérgio Reis Saraiva Pina ${ }^{3}$, \\ Isabel Maria Mousinho de Almeida Galriça Neto ${ }^{4}$, Júlio Almeida ${ }^{5}$
}

Quaresma F, Lérias A, Reis-Pina P, Galriça Neto I, Almeida J. Delirium numa enfermaria de Medicina Interna - impacto na prática clínica / Delirium in internal medicine ward - impact on clinical practice. Rev Med (São Paulo). 2020 jul.-ago.;99(4):357-65.

RESUMO: Introdução: O delirium é frequente nos doentes idosos internados. Define-se como uma alteração aguda e flutuante ao nível da cognição, atenção e consciência, geralmente reversível e de etiologia multifactorial, com aumento da morbimortalidade e dos custos, mas que permanece sub-diagnosticada. Neste sentido, avaliou-se a ocorrência de delirium numa enfermaria de Medicina Interna, os principais factores de risco e o impacto no episódio de internamento e após 3 meses. Materiais e Métodos: estudo prospectivo observacional de 3 meses num serviço de um hospital terciário, com aplicação da escala de sedação e agitação de Richmond adaptada e do Método de Avaliação da Confusão para o rastreio e diagnóstico de delirium, confirmadas pelos critérios do Diagnostic and Statistical Manual of Mental Disorders, $5^{a}$ edição. Avaliaram-se os principais factores de risco, segundo as guidelines, e o impacto no internamento e sobrevida aos 3 meses. Resultados/Discussão: Numa amostra de 297 doentes, a ocorrência de delirium foi de $26 \%$, na sua maioria à admissão $(73 \%)$ e na forma hiperactiva $(50 \%)$. Estes doentes eram mais idosos, com mais comorbilidades, nomeadamente demência, e menos autonomia. Identificou-se a contenção física, a limitação da autonomia e as alterações do potássio como factores independentes para a ocorrência de delirium. Este associou-se a maior mortalidade no internamento e aos 3 meses, com maior sobrecarga dos profissionais de saúde. A aplicação das escalas permitiu um maior reconhecimento desta patologia (12\% para $26 \%$ ), nomeadamente as formas hipoactivas. Conclusão: O delirium foi frequente e com elevado impacto nos doentes e profissionais de saúde.

Descritores: Delírio; Medicina interna; Idoso; Mortalidade.
ABSTRACT: Introduction: Delirium is common in hospitalized elderly patients. It is defined as an acute and fluctuating, usually reversible, alteration of the level of cognition, attention and consciousness, with a multifactorial etiology, associated with increased morbimortality and costs, however, remains underdiagnosed. We evaluated the occurrence of delirium in an Internal Medicine ward, it's main risk factors and consequences during hospitalization and 3 months after discharge. Materials and Methods: prospective 3-month observational study in a ward of a tertiary hospital, using the adaptation of Richmond sedation and agitation scale and the Confusion Assessment Method for the diagnosis of delirium, confirmed by the criteria of the Diagnostic and Statistical Manual of Mental Disorders, $5^{\text {th }}$ edition. The main risk factors were selected according to the guidelines. Hospitalization consequences and survival at 3 months were evaluated. Results/Discussion: In a sample of 297 patients, $26 \%$ presented delirious, mostly at admission (73\%), with a hyperactive phenotype $(50 \%)$. These patients were older, with more comorbidities, namely dementia, and less autonomy. Physical restraint, autonomy impairment, and serum potassium levels were identified as independent factors for delirium. It was also associated with higher in-hospital and 3 months mortality, and major burden on health professionals. The application of the scales allowed a greater recognition of this syndrome (12\% to $26 \%$ ), namely the hypoactive forms. Conclusion: Delirium was frequent and with a high impact on patients and health professionals.

Keywords: Delirium; Internal medicine; Aged; Mortality.

Instituição: Unidade Funcional Medicina 1.2 - H. São José, CHULC.

Apresentação oral no $24^{\circ}$ Congresso Nacional de Medicina Interna, 31 maio de 2018, Algarve - Salgados, Portugal.

1. Assistente Hospitalar de Medicina Interna. Unidade Funcional Medicina 1.2 - H. São José, Centro Hospitalar Universitário de Lisboa (CHULC), Portugal. ORCID: https://orcid.org/0000-0001-7054-1812. Email: Email: fquaresma15@gmail.com.

2. Assistente Hospitalar Graduado de Psiquiatria. Serviço de Psiquiatria de Ligação - H. São José, CHULC, Portugal. ORCID: https://orcid.org/00000003-3941-000X. Email: amelialerias@sapo.pt.

3. Mestre. Faculdade de Medicina da Universidade de Lisboa, Portugal. ORCID: https://orcid.org/0000-0002-4665-585X. Email: preispina@hotmail.com

4. Mestre. Unidade de Cuidados Continuados e Paliativos, Hospital da Luz, Lisboa, Portugal. ORCID: https://orcid.org/0000-0001-8018-9912. Email: isaneto@netcabo.pt.

5. Assistente Hospitalar Graduado Sénior, Director de Serviço. Unidade Funcional Medicina 1.2 - H. São José, CHULC, Portugal. ORCID: https:// orcid.org/0000-0003-4594-9714. Email: julio.almeida@chlc.min-saude.pt.

Endereço para correspondência: Filipa Quaresma. R. José António Serrano, 1150-199. Lisboa, Portugal. Email: fquaresma15@gmail.com. 


\section{INTRODUÇÃO}

Com o aumento da esperança média de vida e envelhecimento global da população, que em Portugal atinge uma das taxas mais elevadas da Europa ${ }^{1,2}$, tem-se observado o inerente aumento da população idosa e das doenças crónicas e irreversíveis. Tal condiciona a necessidade de cuidados específicos e dirigidos, muitas vezes em regime de internamento, o que implica importantes custos para o sistema de saúde ${ }^{3}$.

Entre os problemas mais frequentes nos internamentos destaca-se o delirium, particularmente na população idosa e em fim de vida ${ }^{4}$. É uma síndrome neuropsiquiátrica que se caracteriza por uma alteração aguda e flutuante ao nível da cognição, atenção e consciência, geralmente reversível $^{5}$. Apesar da patofisiologia pouco esclarecida, o delirium parece resultar de uma alteração cerebral aguda, associada a alterações de neurotransmissores (p.ex: défice de acetilcolina ou défice dopaminérgico $)^{6}$, geralmente de etiologia multifactorial ${ }^{4}$. Entre os seus principais factores de risco encontram-se a idade avançada e a demência, ambas a aumentar ${ }^{6,7}$, bem como a infecção, a dor, o uso de psicofármacos, alterações metabólicas, hidroelectrolíticas, entre outros ${ }^{4}$.

É a síndrome neuropsiquiátrica mais frequente em meio hospitalar, uma das seis principais doenças preveníveis no idoso internado, associada a pior prognóstico, e maior número de complicações durante e após o internamento ${ }^{4}$. Efectivamente, o delirium tem um elevado impacto para os cuidados de saúde e a nível sócioeconómico, nomeadamente com aumento da morbimortalidade e dos custos, sendo também uma fonte de sofrimento para o doente e família. ${ }^{4}$ No entanto, apesar do crescente interesse sobre o delirium, este permanece uma entidade pouco compreendida e sub-diagnosticada ${ }^{4}$.

Em Portugal, têm surgido alguns trabalhos publicados na área do delirium, contudo na sua maioria desenvolvidos em tipologias específicas de doentes que não numa enfermaria de Medicina Interna $(\mathrm{EMI})^{8-14}$.

Nesse sentido, os autores pretenderam avaliar a ocorrência de delirium numa EMI e analisar o impacto do uso sistematizado de ferramentas para o seu reconhecimento, bem como identificar os principais factores de risco e consequências associadas a esta entidade e o seu impacto na sobrevivência aos três meses.

\section{MATERIAL E MÉTODOS}

Realizou-se um estudo prospectivo observacional durante três meses (março a junho de 2017) numa EMI de um hospital central e universitário em Lisboa, Portugal. Foram incluídos todos os doentes admitidos de forma consecutiva na EMI com idade superior a 18 anos. Foram excluídos os doentes provenientes de outras enfermarias, que permaneceram menos de 24 horas no internamento, que não falassem português ou que tivessem recusado participar. $\mathrm{O}$ estudo incidiu sobre a primeira semana de internamento (7 dias) dos doentes seleccionados.

Para o rastreio de delirium foi aplicada a escala de sedação e agitação de Richmond adaptada (RASSad) ${ }^{15-16}$, que consiste numa classificação do grau de agitação psicomotora, de -5 a 4 , considerando-se normal os valores entre -1 e 1 . Nos doentes com delirium, os valores superiores a 1 correspondem a quadros hiperactivos, e os inferiores a -1 a hipoactivos. Na presença de graus de agitação que variem entre valores positivos e negativos classificar-seia como episódio misto. A RASSad foi aplicada a todos os doentes admitidos na EMI, sem qualquer critério de exclusão, três vezes ao dia pela equipa de enfermagem (1 vez em cada turno).

Para o diagnóstico de delirium, foi aplicado pelo médico investigador (FQ) a versão traduzida e validada para português do Método de Avaliação da Confusão (CAM $)^{17}$ ao primeiro e sétimo dia de internamento; e também em caso de intercorrências (qualquer avaliação pela Urgência Interna ou se mais de duas avaliações alteradas consecutivas RASSad). Todos os casos foram confirmados pela aplicação do Diagnostic and Statistical Manual of Mental Disorders $5^{\mathrm{a}}$ edição (DSM-5), considerado o gold standard para o diagnóstico de delirium.

Foi considerado delirium à admissão quando presente de início ou nas primeiras $48 \mathrm{~h}$, e delirium durante o internamento (intercorrente), caso surgisse após as $48 \mathrm{~h}$ de internamento no decorrer da primeira semana de internamento.

A identificação do delirium através das escalas foi comparada com os dados de um estudo retrospectivo sobre a sua ocorrência (baseado na referência a este diagnóstico no processo clínico) realizado na mesma EMI num período idêntico no ano prévio e que incluiu 338 doentes.

Não foi obtido um consentimento informado escrito pela natureza não-intervencional do estudo, com um desvio mínimo da prática clínica habitual, e por que dele não decorria nenhum risco para os doentes (Artigo 6 da Convenção de Oviedo), cuja vontade (e dos seus familiares) foi sempre respeitada. Não obstante, sempre que colaborante, foi obtido o consentimento verbal do doente ou do cuidador/familiar presente.

Foram colhidos os dados demográficos (género, idade e local de residência); comorbilidades (número e índice de Charlson) ${ }^{18}$; o performance status ${ }^{19}$, que quantifica o impacto da doença nas actividades de vida diária, numa ordem crescente de incapacidade $(0$ - activo sem restrições, até 5 - morte); o grau de autonomia prévio através da escala de Barthel $^{20}$, que avalia a capacidade de realizar de forma autónoma 10 actividades básicas da vida diária numa escala de 0 (dependente) a 100 (totalmente independente); e o diagnóstico principal de internamento. 
Salienta-se que o diagnóstico de demência foi considerado apenas nos casos em que já estava descrito previamente no processo clínico ou antecedentes pessoais.

Após a revisão da literatura foram selecionados os principais factores descritos como precipitante/ predisponente de delirium $^{4,21-22}$, tendo-se considerado a medicação prévia quanto ao tipo e número de fármacos (polimedicação: uso de 5 ou mais fármacos ${ }^{23}$ ); medicação administrada durante o internamento, instituída previamente à identificação/diagnóstico do episódio de delirium; dados clínicos e laboratoriais quanto a presença de infecção (sintomas e sinais, elevação dos parâmetros inflamatórios, culturas microbiológicas positivas ou prescrição de antibiótico), desidratação (sintomas, sinais e alteração do ratio ureia/creatinina), alterações iónicas ( $135<$ sódio > 145 $\mathrm{mEq}$; $3.5<$ potássio $>5.5 \mathrm{mEq})$ e da glicémia $(<70$ ou $>200$ $\mathrm{mg} / \mathrm{dL}$ ). Avaliou-se ainda, pelos registos de enfermagem, a presença de úlceras de pressão, algaliação, obstipação, padrão de sono (normal, reduzido ou aumentado), quedas ou utilização de contenção física. Todos estes factores foram considerados apenas se presentes previamente à identificação/diagnóstico de delirium.

Procurou-se ainda avaliar as possíveis repercussões e consequências do delirium pelo que se contabilizou a intervenção da equipa de Urgência Interna (número de chamadas), necessidade de contenção farmacológica ou aplicação de medidas não farmacológicas (p.ex: reorientação, camas baixas, etc.); e os pedidos de colaboração de Psiquiatria de Ligação (número de pedidos), a especialidade que na nossa instituição responde a estas intercorrências. Por fim, analisou-se o tempo de internamento na EMI (até à alta, transferência ou morte), destino de alta (considerando-se institucionalização a alta para Lar ou Rede Nacional de Cuidados Continuados), sobrevivência à alta e aos três meses (pela consulta do processo informático e do Registo de Saúde Electrónico RSE) e número de readmissões nesses três meses, segundo a consulta do RSE. A recolha de dados foi realizada de forma prospectiva pela médica investigadora (FQ) directamente com os doentes e/ou através da consulta dos processos clínicos informáticos, registos médicos e de enfermagem, e a RSE.

Procedeu-se à análise descritiva univariada através da mediana, média, desvio padrão, e teste de Mann-Whitney no caso das variáveis contínuas; e de proporções e teste do Qui-quadrado ou de Fisher para as variáveis categóricas; fez-se ainda uma sub-análise ajustada para a idade, dada a maior prevalência de delirium nos idosos ( $>65$ e $>80$ anos). Seguidamente efectuou-se a análise multivariada dos factores de risco para identificar as variáveis com associação independente com o delirium. Utilizou-se um modelo de regressão logística, pelo método de Foward Stepwise, e a qualidade do ajuste do modelo foi validada pelo teste de Hosmer e Lemeshow. O nível de significância estatística adoptado foi de $\mathrm{p}<0.05$ (intervalo de confiança a 95\%). Realizou-se ainda a análise de sobrevivência aos 3 meses após alta (endpoint: mortalidade por qualquer causa), segundo o método de Kaplan-Meier e comparadas pelo teste do log-rank. A análise estatística foi realizada com o software SPSS 22.0®

\section{RESULTADOS}

A amostra final do estudo teve 297 doentes. Verificaram-se $78(26 \%)$ casos de delirium, a maioria presente à admissão $(\mathrm{N}=57,73 \%)$ (Figuras 1 e 2). Destes, $30 \%$ persistiram durante a primeira semana; e observaramse $21(27 \%)$ casos de delirium intercorrente (Figura 2).

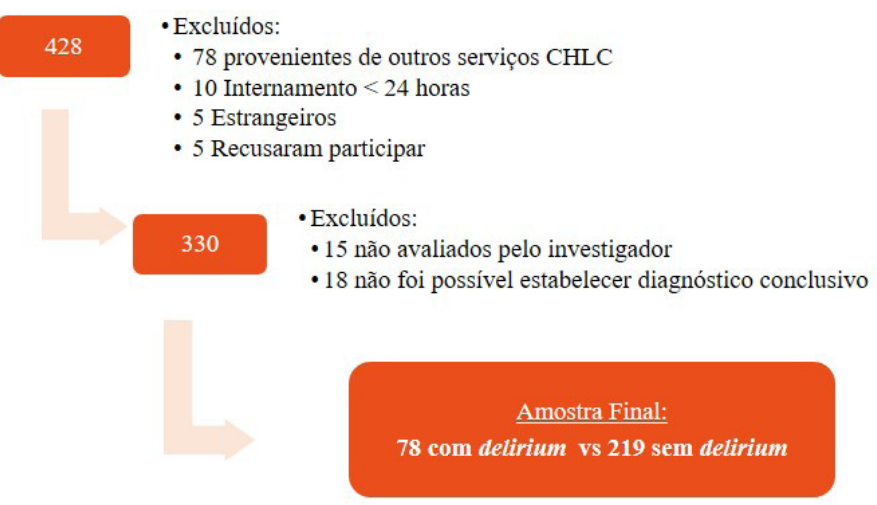

Figura 1 - Diagrama de selecção da amostra do estudo 
Quaresma F, et al. Delirium numa enfermaria de Medicina Interna - impacto na prática clínica.

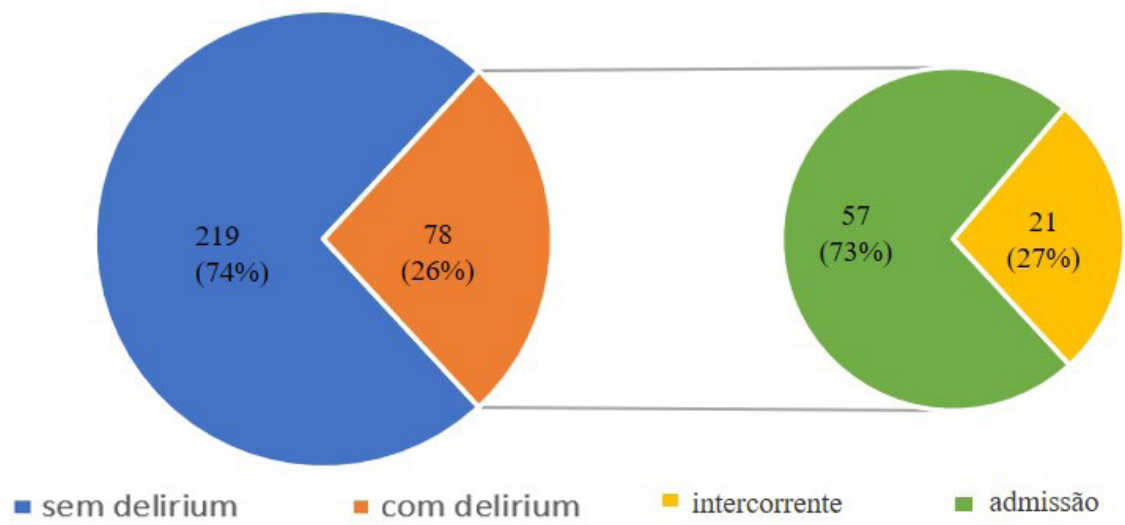

Figura 2 - Ocorrência de delirium quanto ao momento do internamento

$\mathrm{Na}$ amostra, os doentes com delirium eram mais idosos, com maior número de comorbilidades, nomeadamente demência, menos autonomia prévia e apresentavam um maior número de institucionalização prévia ao internamento (Tabela 1).

Tabela 1 - Principais factores de risco da amostra quanto à presença de delirium

\begin{tabular}{|c|c|c|c|c|}
\hline Factores de Risco & $\begin{array}{c}\text { Sem delirium (219) } \\
\text { N (\%) }\end{array}$ & $\begin{array}{c}\text { Com delirium (78) } \\
\text { N (\%) }\end{array}$ & $\begin{array}{l}\text { Univariada } \\
\text { OR (IC 95\%) } \\
\text { Valor-p** }\end{array}$ & $\begin{array}{l}\text { Multivariada } \\
\text { OR (IC 95\%) } \\
\text { Valor-p*** }\end{array}$ \\
\hline Idade (média \pm D.P.) & $73 \pm 15.11$ & $82 \pm 10.43$ & $<0.0001^{\mathrm{a}}$ & \\
\hline $\begin{array}{l}\text { Feminino } \\
\text { Masculino }\end{array}$ & $\begin{array}{l}117(53) \\
102(47)\end{array}$ & $\begin{array}{l}47(60) \\
31(40)\end{array}$ & $\begin{array}{c}0.76(0.45-1.28) \\
0.297^{\mathrm{b}}\end{array}$ & \\
\hline $\mathbf{N}^{0}$ comorbilidades (média \pm D.P.) & $7 \pm 3.06$ & $6 \pm 2.45$ & $0.023^{\mathrm{a}}$ & \\
\hline Índ. de Charlson (mediana) & 6 & 6 & $0.324^{\mathrm{a}}$ & \\
\hline Demência & $27(12)$ & $29(37)$ & $\begin{array}{c}4.21(2.29-7.75) \\
<\mathbf{0 . 0 0 0 1}^{\mathrm{b}}\end{array}$ & \\
\hline $\begin{array}{l}\text { Residência } \\
\text { Domićíiio } \\
\text { Instituição Apoio Social } \\
\text { Outra }\end{array}$ & $\begin{array}{l}199(91) \\
18(8) \\
2(1)\end{array}$ & $\begin{array}{l}63(81) \\
13(17) \\
2(2)\end{array}$ & $\begin{array}{c}0.42(0.20-0.87) \\
\mathbf{0 . 0 1 8}^{\mathbf{b}}\end{array}$ & \\
\hline $\begin{array}{l}\text { Índice de Barthel (mediana) } \\
\text { Performance Status (mediana) }\end{array}$ & $\begin{array}{c}90 \\
1\end{array}$ & $\begin{array}{l}50 \\
2\end{array}$ & $\begin{array}{l}<0.0001^{\mathrm{a}} \\
<0.0001^{\mathrm{a}}\end{array}$ & $\begin{array}{c}0.97(0.96-0.99) \\
\quad<\mathbf{0 . 0 0 0 1}\end{array}$ \\
\hline Polimedicação & $140(64)$ & $48(62)$ & $\begin{array}{c}0.90(0.53-1.54) \\
0.707^{\mathrm{b}}\end{array}$ & \\
\hline $\begin{array}{l}\text { Fármacos de risco } \\
\text { média } \pm \text { D.P (min-max) }\end{array}$ & $2 \pm 1.76(0-9)$ & $3 \pm 1.94(0-8)$ & $\begin{array}{c}0.99(0.48-2.02) \\
0.977^{\mathrm{b}}\end{array}$ & \\
\hline Opióides & $27(12)$ & $12(15)$ & $\begin{array}{c}0.77(0.37-1.61) \\
0.493^{\mathrm{b}}\end{array}$ & \\
\hline Corticosteroides & $46(21)$ & $14(18)$ & $\begin{array}{c}1.22(0.63-2.36) \\
0.564^{\mathrm{b}}\end{array}$ & \\
\hline Benzodiazepinas & $98(45)$ & $30(38)$ & $\begin{array}{c}1.30(0.76-2.20) \\
0.336^{\mathrm{b}}\end{array}$ & \\
\hline Antipsicóticos & 41 (19) & $46(59)$ & $\begin{array}{c}0.16(0.09-0.28) \\
<\mathbf{0 . 0 0 0 1}^{\mathbf{b}}\end{array}$ & \\
\hline Antihistamínicos & $19(9)$ & $8(10)$ & $\begin{array}{c}0.83(0.35-1.98) \\
0.677^{\mathrm{b}}\end{array}$ & \\
\hline Antieméticos & $35(16)$ & $7(9)$ & $\begin{array}{c}1.93(0.82-4.54) \\
0.127^{\mathrm{b}}\end{array}$ & \\
\hline Antidepressivos & $31(14)$ & $9(12)$ & $\begin{array}{c}1.26(0.57-2.79) \\
0.561^{\mathrm{b}}\end{array}$ & \\
\hline Antiparkinsónicos & $6(3)$ & $7(9)$ & $\begin{array}{c}0.29(0.09-0.88) \\
0.046^{\mathrm{C}}\end{array}$ & \\
\hline Anticonvulsivantes & $23(11)$ & $10(13)$ & $\begin{array}{c}0.80(0.36-1.76) \\
0.576^{\mathrm{b}}\end{array}$ & \\
\hline Outros psicofármacos & $28(13)$ & $6(8)$ & $\begin{array}{c}1.76(0.70-4.43) \\
0.225^{\mathrm{b}} \\
\end{array}$ & \\
\hline
\end{tabular}


Tabela 1 - Principais factores de risco da amostra quanto à presença de delirium

continuação

\begin{tabular}{|c|c|c|c|c|}
\hline Factores de Risco & $\begin{array}{c}\text { Sem delirium (219) } \\
\mathrm{N}(\%)\end{array}$ & $\begin{array}{c}\text { Com delirium (78) } \\
\text { N (\%) }\end{array}$ & $\begin{array}{c}\text { Univariada } \\
\text { OR (IC 95\%) } \\
\text { Valor-p* } \\
\end{array}$ & $\begin{array}{c}\text { Multivariada } \\
\text { OR (IC 95\%) } \\
\text { Valor-p** }\end{array}$ \\
\hline Infecção & $109(50)$ & $58(74)$ & $\begin{array}{c}2.93(1.65-5.19) \\
<\mathbf{0 . 0 0 0 1}^{\mathbf{b}}\end{array}$ & \\
\hline Desidratação & $103(47)$ & $40(51)$ & $\begin{array}{c}1.19(0.71-1.99) \\
0.519^{\mathrm{b}}\end{array}$ & \\
\hline Obstipação & $59(27)$ & $33(42)$ & $\begin{array}{c}1.99(1.16-3.41) \\
\mathbf{0 . 0 1 2}^{\mathbf{b}}\end{array}$ & \\
\hline Úlcera de pressão & $12(5)$ & $19(24)$ & $\begin{array}{c}5.56(2.55-12.10) \\
\quad<\mathbf{0 . 0 0 0 1}^{\mathbf{b}}\end{array}$ & \\
\hline Algaliação & $45(21)$ & $28(36)$ & $\begin{array}{c}2.21(1.25-3.90) \\
\mathbf{0 . 0 0 6}^{\mathbf{b}}\end{array}$ & \\
\hline Padrão sono alterado & $87(40)$ & $41(53)$ & $\begin{array}{c}2.31(1.27-4.21) \\
\mathbf{0 . 0 0 5}^{\mathbf{b}}\end{array}$ & \\
\hline Queda & $6(3)$ & $5(6)$ & $\begin{array}{c}2.43(0.72-8.21) \\
0.140^{c}\end{array}$ & \\
\hline Contenção física & $9(4)$ & $41(53)$ & $\begin{array}{c}25.86(11.6-57.64) \\
<\mathbf{0 . 0 0 0 1}^{\mathbf{c}}\end{array}$ & $\begin{array}{c}68.59(16.37-287.5) \\
<\mathbf{0 . 0 0 0 1}\end{array}$ \\
\hline $\begin{array}{l}\text { Alterações iónicas } \\
\text { - Sódio } \\
\text { - Potássio }\end{array}$ & $\begin{array}{l}71(32) \\
48(22) \\
38(17)\end{array}$ & $\begin{array}{l}43(55) \\
27(35) \\
23(29)\end{array}$ & $\begin{array}{c}2.49(1.47-4.23) \\
\mathbf{0 . 0 0 1}^{\mathbf{b}} \\
1.85(1.05-3.26) \\
\mathbf{0 . 0 3 1}^{\mathbf{b}} \\
1.92(1.05-3.49) \\
\mathbf{0 . 0 3 2}^{\mathbf{b}}\end{array}$ & $\begin{array}{c}3.64 ;(1.12-11.85) \\
\mathbf{0 . 0 3 2}\end{array}$ \\
\hline Alterações da glicémia & $18(8)$ & $14(18)$ & $\begin{array}{c}2.93(1.32-6.48) \\
\mathbf{0 . 0 0 6}^{\mathbf{b}}\end{array}$ & \\
\hline
\end{tabular}

* valores com significado estatístico estão assinalados a negrito; **T. Hosmer e Lemeshow p=0.731; a) Teste de Mann-Whitney; b) Teste do Quiquadrado; c) Teste de Fisher.

Os diagnósticos principais mais frequentes foram do grupo nosológico cardiovascular e respiratório $(29 \%, \mathrm{~N}=97$ e $24 \%, N=79$ respectivamente), com elevada prevalência de infecção, independentemente do foro da patologia $(43 \%$, $\mathrm{N}=143$ ).

Quanto ao fenótipo psicomotor, a forma hiperactiva foi a mais frequente $(\mathrm{N}=39,50 \%)$, assinalando-se, contudo, que, nos casos à admissão, prevaleceram as formas hipoactivas ( $\mathrm{N}=30,53 \%)$ (Figura 3 ).

Destaca-se a associação significativa entre o delirium e a limitação da autonomia $(\mathrm{p}<0.0001, \mathrm{OR}=0.97$, IC 95\% 0.96-0.99), a aplicação de medidas de contenção física $(\mathrm{p}<0.0001, \mathrm{OR}=68.59$, IC 95\% 16.37-287.5), e as alterações do potássio $(\mathrm{p}=0.032 ; \mathrm{OR}=3.64$, IC 95\% 1.1211.85), segundo a análise multivariada (Tabela 1).

A necessidade de avaliação pela Urgência Interna e pela Psiquiatria de Ligação foi mais elevada no grupo com delirium (Tabela 2) e associou-se a maior utilização de terapêutica farmacológica, sendo um possível indicador da maior sobrecarga dos profissionais de saúde nesta patologia. Observou-se ainda que esta entidade foi mais frequente entre os doentes paliativos $(\mathrm{N}=17 / 35 ; \mathrm{p}<0.0001)$, nomeadamente naqueles em fim de vida $(\mathrm{N}=13 / 19$, $\mathrm{p}>0.0001)$.

Houve tendência para um maior tempo de internamento na amostra com delirium, mas sem significado estatístico $(\mathrm{p}=0.055)$; com uma mortalidade no internamento quase 3 vezes superior, e 2 vezes mais elevada aos 3 meses após alta neste grupo (Tabela 2 e Figura 4).

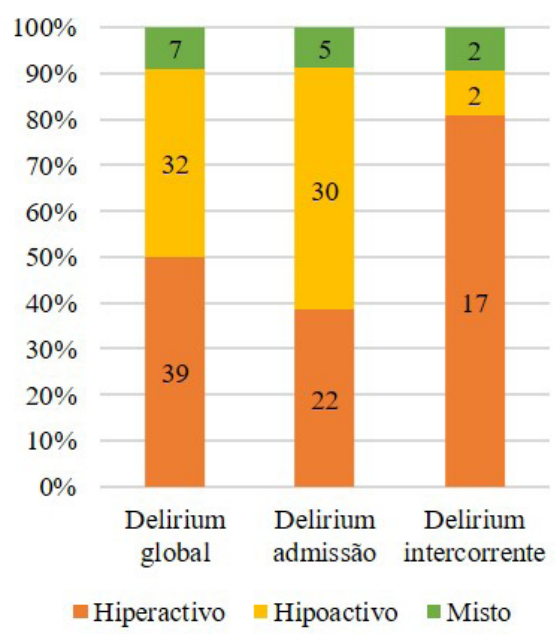

Figura 3 - Delirium quanto ao fenótipo psicomotor (RASSad) 
Quaresma F, et al. Delirium numa enfermaria de Medicina Interna - impacto na prática clínica.

Tabela 2 - Consequências no internamento e aos 3 meses na amostra com e sem delirium

\begin{tabular}{|c|c|c|c|c|}
\hline Consequências & $\begin{array}{c}\text { Sem delirium (219) } \\
\text { N (\%) }\end{array}$ & $\begin{array}{c}\text { Com delirium (78) } \\
\text { N (\%) }\end{array}$ & OR (IC 95\%) & Valor-p* \\
\hline Urgência Interna & $20(9)$ & $36(46)$ & $8.53(4.50-16.17)$ & $<0.0001^{b}$ \\
\hline Contenção Farmacológica & $9(4)$ & $35(45)$ & $18.99(8.51-42.38)$ & $<0.0001^{\text {b }}$ \\
\hline Psiquiatria de Ligação & $9(4)$ & $13(17)$ & $4.67(1.91-11.41)$ & $<0.0001^{\text {b }}$ \\
\hline $\begin{array}{l}\text { Tempo internamento - dias } \\
\text { (média } \pm \text { D.P.) }\end{array}$ & $11 \pm 9.62$ & $13 \pm 11.66$ & & $0.055^{\mathrm{a}}$ \\
\hline $\begin{array}{l}\text { Destino de alta } \\
\text { (sem óbitos no internamento) } \\
\text { Transferência } \\
\text { Manteve } \\
\text { Alterado }\end{array}$ & $\begin{array}{c}19(9) \\
184(84) \\
10(4)\end{array}$ & $\begin{array}{c}6(8) \\
55(70) \\
4(5)\end{array}$ & $0.87(0.40-1.89)$ & $0.719^{b}$ \\
\hline Óbito internamento & $6(3)$ & $13(17)$ & $7.1(2.60-19.42)$ & $<0.0001^{\mathrm{c}}$ \\
\hline Sobrevida 3 meses & $189(86)$ & $54(69)$ & $3.1(1.67-5.83)$ & $<0.0001^{\text {b }}$ \\
\hline Readmissão 3 meses & $72(33)$ & $30(38)$ & $0.89(0.49-1.61)$ & $0.695^{\mathrm{b}}$ \\
\hline
\end{tabular}

* valores com significado estatístico estão assinalados a negrito; a) Teste de Mann-Whitney; b) Teste do Qui-quadrado; c) Teste de Fisher
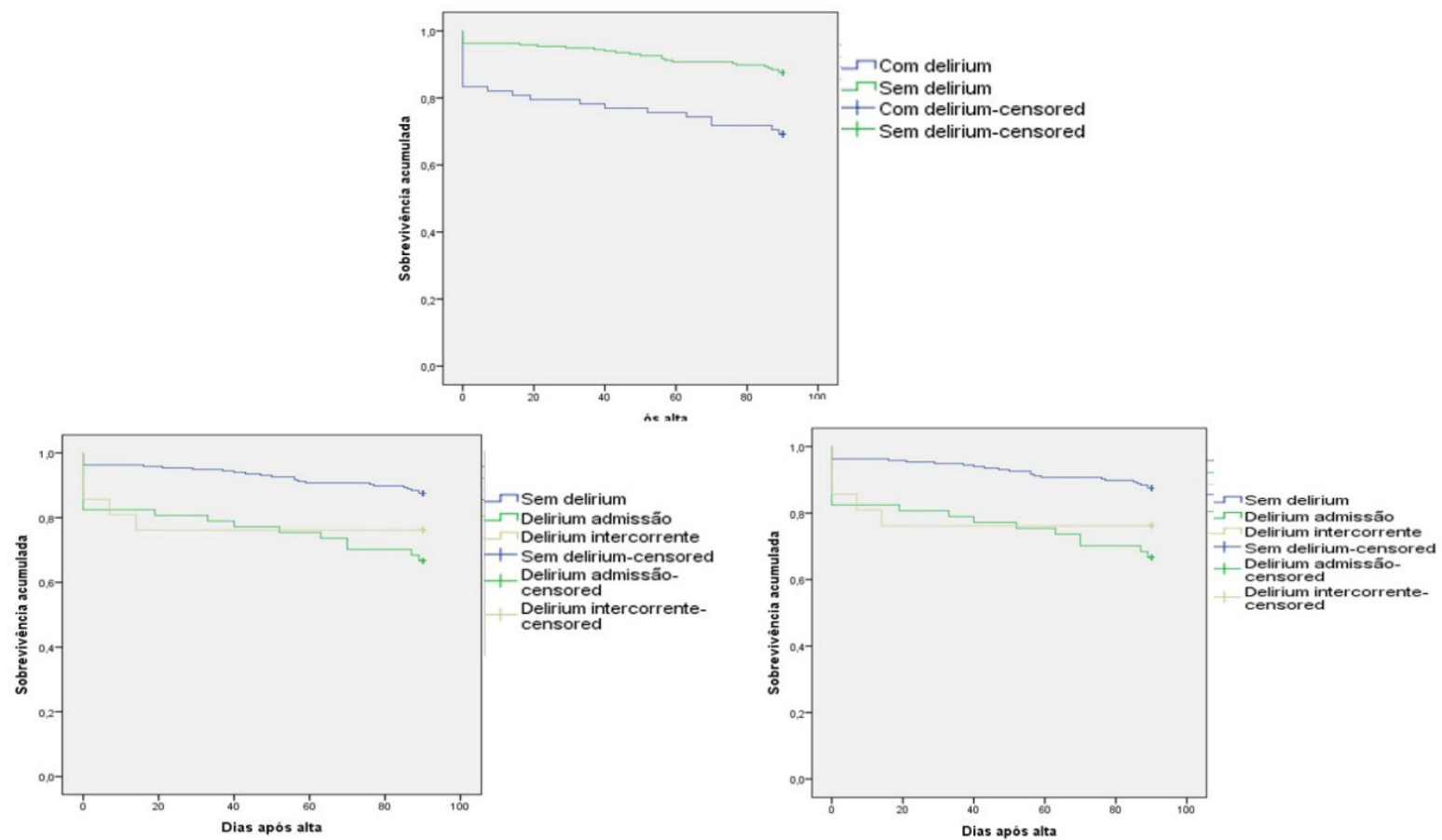

Figura 4 - Curvas de sobrevivência aos 3 meses para a ocorrência de delirium global, quanto ao momento do internamento e quanto ao fenótipo psicomotor

\section{DISCUSSÃO}

Numa amostra de 297 doentes, a ocorrência de delirium foi de $26 \%$, na sua maioria à admissão $(73 \%)$ e na forma hiperactiva (50\%). Estes doentes eram mais idosos, com mais comorbilidades, nomeadamente demência prévia, e menos autonomia. Identificou-se a contenção física, a limitação da autonomia e as alterações do potássio como factores independentes para a ocorrência de delirium. Este associou-se a maior mortalidade no internamento e aos 3 meses, bem como com uma maior sobrecarga dos profissionais de saúde. A aplicação das escalas permitiu um maior reconhecimento desta patologia (12\% para $26 \%$ ), nomeadamente as formas hipoactivas. 
Efectivamente, este trabalho corroborou a evidência prévia de que o delirium é uma patologia frequente que continua subdiagnosticada e cujo reconhecimento pode ser melhorado com a implementação de escalas de diagnóstico ${ }^{24-25}$. De facto, a sua aplicação permitiu uma franca optimização da identificação do delirium, pois, comparativamente ao estudo retrospectivo na mesma EMI, identificou uma incidência de delirium de $26 \%$ no estudo prospectivo, comparativamente a $12 \%$ no ano prévio.

Esta incidência encontra-se de acordo com o descrito na literatura $(11-34 \%)^{4,26}$, e foi semelhante a um trabalho realizado em Portugal (apesar de realizado num Serviço de Urgência), com uma incidência de $20 \%{ }^{10}$.

Verificou-se também um predomínio do delirium à admissão ( $\mathrm{N}=57 ; 73 \%)$, sobreponível ao descrito em alguns estudos, apesar dos resultados publicados serem um pouco discrepantes ${ }^{27}$. O elevado número de casos à admissão pode decorrer de diversos factores, destacando-se que neste Centro Hospitalar muitos doentes permanecem algum tempo na Urgência antes da admissão na EMI, o que pode potenciar o desenvolvimento de delirium, nomeadamente pelas mudanças frequentes de cama/localização do doente; ser um ambiente sem rotinas estabelecidas e em que as condições ambientais (p.ex. luminosidade, ruído, referências temporais como relógios etc) são pouco favoráveis ${ }^{28}$.

Quanto ao fenótipo psicomotor, a aplicação das escalas também permitiu a sua melhor caracterização, nomeadamente das formas hipoactivas (retrospectivo $2 \%, \mathrm{~N}=1 / 41$ vs. prospectivo $41 \%, \mathrm{~N}=32 / 78$ ), descritas como mais frequentes, mas menos reconhecidas, e que se associam a um pior prognóstico ${ }^{4,29-30}$. Não obstante, a forma psicomotora de delirium mais frequentemente identificada neste estudo foi a hiperactiva $(50 \%)$.

Entre os factores associados ao delirium, o internamento, que foi comum a todos os casos, é por si só uma importante alteração ambiental e está bem estabelecido como precipitante desta patologia ${ }^{4,28,31}$. Entre os outros elementos analisados, verificou-se uma forte associação com a contenção física, com um risco 5.5 vezes superior de apresentar delirium. Apesar de ser uma prática contraindicada, com recomendações claras da Direção Geral de Saúde ${ }^{32}$, é ainda frequente nas enfermarias, alegando promover uma maior segurança para o doente. Está ainda bem documentada como factor para o desenvolvimento e persistência de delirium nos doentes internados ${ }^{4,22,28,33}$. Também os efeitos dos fármacos são precipitantes bem estabelecidos ${ }^{4,34}$, tendo algumas classes um maior risco por actuarem ao nível das vias patofisiológicas envolvidas no delirium, particularmente as vias colinérgicas e dopaminérgicas $^{34-35}$. O uso de antipsicóticos foi um factor independentemente relacionado com a ocorrência de delirium hiperactivo, mesmo após ajuste para a idade; contudo, pela metodologia deste estudo é difícil identificar a razão da prescrição e discernir se este foi um precipitante ou se apenas é uma classe frequentemente prescrita. Os antipsicóticos pela sua acção ao nível dos receptores dopaminérgicos, reduzindo a dopamina, têm sido apontados para o tratamento do delirium, apesar de alguma inconsistência em termos de evidência científica ${ }^{34-36}$.

As alterações ao nível dos iões, como o sódio e potássio, e também da glicémia, têm sido associadas ao delirium, como se verificou neste trabalho (Tabela 1). Apesar de ser necessária mais evidência científica para estabelecer esta relação $0^{4,28}$, considera-se que resulte do papel destes elementos em algumas das principais vias fisiopatológicas envolvidas na génese do delirium ${ }^{4,35}$.

Foi claro o impacto do delirium, quer ao nível da sobrecarga dos profissionais de saúde, quer para a sobrevida dos doentes, como previamente documentado ${ }^{4,26-28,35}$.

Alguns autores defendem que a mortalidade se deve ao delirium propriamente dito $^{21}$, no entanto não é claro se não é a consequência dos factores subjacentes do doente que conduzem à morte e também o tornaram mais predisposto ao delirium. Verificou-se que, na sua maioria, os óbitos ocorreram de forma precoce, na primeira semana de internamento, o que traduz a gravidade dos quadros clínicos nestes doentes.

Assinala-se como pontos fortes deste trabalho o elevado número de doentes analisados; a aplicação sistematizada das escalas para a identificação de delirium superior a $85 \%$; bem como a mais valia da utilização de testes cognitivos numa tipologia em que os doentes têm elevado risco de alterações cognitivas que muitas vezes não estão identificadas ${ }^{37}$. Além do mais, as ferramentas utilizadas foram simples e rápidas de aplicar, com elevada especificidade (>97\%), não implicando grande desvio da prática clínica habitual, pelo que se admite que poderão ser integradas na rotina diária sem aumento significativo da sobrecarga de trabalho. Entre as principais limitações identificadas, destaca-se o facto das escalas de diagnóstico de delirium terem sido aplicadas apenas em dois momentos (excepto se intercorrência) e por um único investigador. Contudo, a taxa de ocorrência de delirium, os factores de risco e a associação com a mortalidade estão de acordo com estudos prévios, sugerindo que a realidade desta EMI portuguesa será semelhante à desses trabalhos. Este foi o primeiro trabalho sobre delirium realizado neste Centro Hospitalar e, da pesquisa realizada, o primeiro também numa EMI. Por ser um estudo unicêntrico, não permite a generalização dos resultados obtidos; contudo, os dados deste estudo são importantes para a necessidade de sensibilizar todos os médicos e outros profissionais de saúde para a valorização e reconhecimento do delirium, do seu impacto neste tipo de enfermarias, e para que se possa melhorar a prática clínica e cuidados aos doentes internados.

Destaca-se a necessidade de formação dos profissionais para melhorar o reconhecimento e prevenção do delirium, através de abordagens multidisciplinares 
Quaresma F, et al. Delirium numa enfermaria de Medicina Interna - impacto na prática clínica.

com optimização das medidas não farmacológicas e da contenção farmacológica, de forma a evitar a contenção física e reduzir as consequências negativas desta entidade no doente, na família e nos profissionais de saúde.

\section{CONCLUSÃO}

O delirium é frequente e tem elevado impacto numa
EMI, cuja população é particularmente susceptível pelo seu marcado envelhecimento, com multimorbilidade e fragilidade inerente. A utilização sistematizada de escalas de rastreio e diagnóstico aumenta a identificação precoce do delirium, permitindo reduzir as suas consequências, nomeadamente ao nível da mortalidade, pelo que urge mudar e melhorar práticas incorrectas.

Agradecimentos: A toda a equipa de Enfermagem desta Unidade Funcional pela sua dedicação, empenho e colaboração na aplicação da escala, e todo o apoio prestado durante a realização deste estudo.

Participação Autores: Filipa Anaia Quaresma: investigadora principal que desenvolveu o trabalho e o executou. Amélia Lérias: Supervisionou o projecto e deu formação na área das escalas para a sua correcta aplicação e execução. Paulo Reis-Pina: orientador da tese de mestrado em que se incluiu este trabalho, supervisionou o desenho do estudo, bem como a redacção do artigo. Isabel Neto: orientador da tese de mestrado em que se incluiu este trabalho, supervisionou o desenho do estudo, bem como a redacção do artigo. Júlio Almeida: Diretor do Serviço em que decorreu o estudo e supervisionou a sua execução e a redacção deste artigo.

\section{REFERÊNCIAS}

1. Organization for Economic Co-operation and Development. Health at a Glance 2017: OECD Indicators. Paris: OECD Publishing; 2017. https://doi.org/10.1787/health_glance2017-en.

2. Lisboa. Instituto Nacional de Estatística. Estatísticas demográficas 2016. Lisboa: INE, IP; 2017.

3. World Health Organization. Preventing chronic diseases: a vital investment. WHO global report. Geneva: WHO; 2005 [cited April 2018]. Available from: http://www.who.int/chp/ chronic_disease_report/en/.

4. Inouye S, Westendorp R, Saczynski J. Delirium in elderly people. Lancet. 2014;383(9920):911-22. doi: https://doi. org/10.1016/S0140-6736(13)60688-1.

5. American Psychiatric Association. Diagnostic and Statistical Manual of Mental Disorders. 5th ed. Washington, DC: American Psychiatric Association; 2013. https://doi. org/10.1176/appi.books.9780890425596.

6. Maldonado JR. Acute brain failure: pathophysiology, diagnosis, management, and sequelae of delirium. Crit Care Clin. 2017;33(3):461-519. doi: https://doi.org/10.1016/j. ccc.2017.03.013.

7. Jacqmin-Gadda H, Alperovitch A, Montlahuc C, et al. 20-Year prevalence projections for dementia and impact of preventive policy about risk factors. Eur J Epidemiol 2013;28:493-502. doi: https://doi.org/10.1007/s10654-013-9818-7.

8. Alvarez-Perez F, Paiva F. Prevalence and risk factors for delirium in acute stroke patients. A retrospective 5-years clinical series. J Stroke Cerebrovasc Dis. 2017;26(3):567-73. doi: https://doi.org/10.1016/j.jstrokecerebrovasdis.2016.11.120.

9. Martins S, Paiva J, Simões M, Fernandes L. Delirium in elderly patients: association with educational attainment. Acta Neuropsychiatr. 2017;29(2):95-101. doi: https://doi. org/10.1017/neu.2016.40.

10. Mariz J, Santos N, Afonso H, Rodrigues P, Faria A, Sousa N, et al. Risk and clinical-outcome indicators of delirium in an Emergency Department Intermediate Care Unit (EDIMCU): an observational prospective study. BMC Emerg Med. 2013;13:2. https://doi.org/10.1186/1471-227X-13-2.
11. Goncalves F, Almeida A, Antunes L, Teixeira S, Pereira $\mathrm{S}$, Edra N. Variation in the incidence of agitated delirium during the day in a palliative care unit: a preliminary report. Am J Hosp Palliat Care. 2013;30(2):111-3. https://doi. org/10.1177/1049909112444302.

12. Cerejeira J, Batista P, Nogueira V, Firmino H, Vaz-Serra A, Mukaetova-Ladinska E. Low preoperative plasma cholinesterase activity as a risk marker of postoperative delirium in elderly patients. Age Ageing. 2011;40:621-6. doi: https://doi.org/10.3389/fphar.2014.00075.

13. Abelha FJ, Botelho M, Fernandes V, Santos A, Dias I, Barros H. Evaluation of delirium in postoperative patients. Arq Med. 2010;24(4):121-8. doi: https://doi.org/10.1016/j. bjane.2012.09.003.

14. Caeiro L, Ferro J, Albuquerque R, Figueira M. Delirium in the first days of acute stroke. J Neurol. 2004;251(2):171-8. https://doi.org/10.1007/s00415-004-0294-6.

15. Chester JG, Beth Harrington M, Rudolph JL; VA Delirium Working Group. Serial administration of a modified Richmond Agitation and Sedation Scale for delirium screening. J Hosp Med. 2012;7(5):450-3. doi: https://doi.org/10.1002/jhm.1003.

16. Nassar Junior AP, Pires Neto RC, de Figueiredo WB, Park M. Validity, reliability and applicability of Portuguese versions of sedation-agitation scales among critically ill patients. Sao Paulo Med J. 2008;126(4):215-9. https://doi.org/10.1590/ S1516-31802008000400003.

17. Sampaio F, Sequeira C. Confusion Assessment Method: tradução e validação para a População Portuguesa dissertação]. Porto: Universidade do Porto; 2012. http:// dx.doi.org/10.12707/RIII12127.

18. Charlson M, Szatrowski TP, Peterson J, Gold J. Validation of a combined comorbidity index. J Clin Epidemiol. 1994;47:1245-51. doi: https://doi.org/10.1016/08954356(94)90129-5.

19. Oken M, Creech R, Tormey D, et al. Toxicity and response criteria of the Eastern Cooperative Oncology Group. Am J Clin Oncol. 1982;5:649-55.

20. Mahoney FI, Barthel DW. Functional evaluation: the Barthel index. Maryland State Med J. 1965;14:61-5.

21. Lawlor PG, Gagnon B, Mancini IL, et al.: Occurrence, causes, 
and outcome of delirium in patients with advanced cancer: a prospective study. Arch Intern Med. 2000;160:786-94. doi: https://doi.org/10.1001/archinte.160.6.786.

22. Inouye SK, Charpentier PA. Precipitating factors for delirium in hospitalized elderly persons. Predictive model and interrelationship with baseline vulnerability. JAMA. 1996;275(11):852-7. doi: https://doi.org/10.1001/ jama.1996.03530350034031.

23. Frazier SC. Health outcomes and polypharmacy in elderly individuals: an integrated literature review. Gerontol Nurs. 2005;31(9):4-11. doi: https://doi.org/10.3928/0098-913420050901-04.

24. Inouye SK, Foreman MD, Mion LC, Katz KH, Cooney LMJr. Nurses' recognition of delirium and its symptoms: comparison of nurse and researcher ratings. Arch Intern Med. 2001;161(20):2467-73. doi: https://doi.org/10.1001/ archinte.161.20.2467.

25. Clegg A, Westby M, Young JB. Under-reporting of delirium in the NHS. Age Ageing 2011;40:283-6. doi: https://doi. org/10.1093/ageing/afq157.

26. Siddiqi N, House AO, Holmes JD. Occurrence and outcome of delirium in medical in-patients: a systematic literature review. Age Ageing. 2006;35(4):350-64. https://doi.org/10.1093/ ageing/afl005.

27. Pendlebury ST, Lovett NG, Smith SC, Dutta N, Bendon C, Lloyd-Lavery A, et al. Observational, longitudinal study of delirium in consecutive unselected acute medical admissions: age-specific rates and associated factors, mortality and readmission. BMJ Open. 2015;5(11):e007808-e007808. doi: https://doi.org/10.1136/bmjopen-2015-007808.

28. National Institute for Health and Clinical Excellence (NICE). National Clinical Guideline Centre. Delirium: diagnosis, prevention and management. Lonon; 2010 [cited April 2018]. Available from: https://www.nice.org.uk/nicemedia/ live/13060/49908/49908.pdf.
29. Meagher D. Motor subtypes of delirium: past, present and future. Int Rev Psychiatry. 2009;21:59-73. doi: https://doi. org/10.1080/09540260802675460.

30. Kim SY, Kim SW, Kim JM, et al. Differential associations between delirium and mortality according to delirium subtype and age: a prospective cohort study. Psychosom Med. 2015;77(8):903-10. doi: https://doi.org/10.1097/ PSY.0000000000000239.

31. Inouye SK. Delirium in older persons. N Engl J Med. 2006;354(11):1157-65. doi: https://doi.org/10.1056/ NEJMra052321.

32. Direcção Geral de Saúde. Orientação n ${ }^{\circ}$ 021/2011: Prevenção de comportamentos dos doentes que põem em causa a sua segurança ou da sua envolvente. Lisboa: DGS; 2011.

33. Tolson D, Morley JE. Physical restraints: abusive and harmful. JAMDA. 2012;13:311-3. doi: https://doi.org/10.1016/j. jamda.2012.02.004.

34. Clegg A, Young JB. Which medications to avoid in people at risk of delirium: a systematic review. Age Ageing. 2011;40(1):23-9. doi: https://doi.org/10.1093/ageing/afq140.

35. Maldonado JR. Neuropathogenesis of delirium: review of current etiologic theories and common pathways. Am J Geriatr Psychiatry. 2013;21:1190-222. doi: https://doi.org/10.1016/j. jagp.2013.09.005.

36. Siddiqi N, Harrison JK, Clegg A, et al. Interventions for preventing delirium in hospitalised non-ICU patients. Cochrane Database Syst Rev. 2016;3:CD005563. doi: https:// doi.org/10.1002/14651858.CD005563.pub3.

37. Sampson EL, Blanchard MR, Jones L, et al. Dementia in the acute hospital: prospective cohort study of prevalence and mortality. Br J Psychiatry. 2009;195:61-6. doi: https://doi. org/10.1192/bjp.bp.108.055335.

Submetido: 18.08 .2019

Aceito: 16.07.2020 